The first hint that Src kinases may have a role in the pathogenesis of Bcr-Abl-driven leukemia came from the observation that Lyn and Hck are activated in a complex with BcrAbl in myeloid cells ${ }^{7,8}$. Hck complexed with Bcr-Abl directly phosphorylates STAT5 (ref. 9) and Tyr177 of $\mathrm{Bcr}-\mathrm{Abl}^{8}$, a residue crucial for the induction of myeloid leukemia in mice. In view of these data, Src kinases seemed to be more important for myeloid than lymphoid transformation, and the results of Hu et al. ${ }^{1}$ caution against placing too much weight on data from cell lines. But we might not yet have the full story with respect to CML. Although Lyn, Hck and Fgr are dispensable, a role for other Src kinases cannot be ruled out, as this would require proof that CGP76030 completely blocks all Src kinases in the CML mice.

Regardless, the authors' work may have important implications for the treatment of $B C R-A B L 1$-positive leukemias. Many individuals with $\mathrm{Ph}^{+}$ALL develop resistance to the Abl kinase inhibitor, imatinib, as a result of mutations in the $B C R-A B L 1$ kinase domain. Given that Src kinases are downstream of Bcr$\mathrm{Abl}$, these individuals should still be responsive to Src inhibitors. In addition, activation of Src kinases has been seen in some resistant individuals without $B C R-A B L 1$ mutations ${ }^{10}$; shutting this 'back door' from the beginning may help prevent this type of resistance.

\section{Exploring B-cell development}

A consistent feature of mouse and human $B C R-A B L 1$-positive $\mathrm{B}-\mathrm{ALL}$ is their pre- $\mathrm{B}$ phenotype, with immunoglobulin heavy but not light chains rearranged ${ }^{11}$. In contrast, the Philadelphia chromosome is rare in pre-preB-ALL or in more mature B-cell malignancies. Thus, it is conceivable that only pre-B-cells have all the 'wiring' in place to be transformed by Bcr-Abl, and that the three Src kinases are required for differentiation to the pre-B stage. Although no B-cell defect has been observed in the triply deficient mice used by Hu et al. ${ }^{1}$, their results may justify a detailed study. Defective B-cell development with arrest at the pro-B stage was reported in $\mathrm{Lyn}^{-/-} \mathrm{Blk}^{-/-} \mathrm{Fyn}^{-/-}$mice ${ }^{12}$. These mice might also be resistant to induction of B-ALL by $\mathrm{Bcr}-\mathrm{Abl}$. Alternatively, $\mathrm{Bcr}-\mathrm{Abl}$ may require Src kinases to arrest B-cells at the pre-B stage.

Another question relates to lymphoid transformation of CML. Analysis of proviral integration sites in mice with both CML and ALL is consistent with independent clones ${ }^{13}$, whereas lymphoid blast crisis arises from the same clone that produced the chronic phase of disease. In chronic-phase CML, a considerable proportion of $\mathrm{B}$-cell progenitors and peripheral blood B-cells are Bcr-Abl-positive ${ }^{14}$ but do not expand. It would be interesting to see if there are also $\mathrm{Ph}^{+}$pre-B-cells. If so, this would argue that an additional genetic event is required to start proliferation, perhaps in contrast to genuine $\mathrm{Ph}^{+} \mathrm{B}-\mathrm{ALL}$. As the work of $\mathrm{Hu}$ et al. ${ }^{1}$ shows, further defining cell type-specific signaling pathways may allow us to develop rational therapies to exploit them.

1. Hu, Y. et al. Nat. Genet. 36, 453-461 (2004).

2. Nowell, P. \& Hungerford, D. Science 132, 1497 (1960).

3. Propp, S. \& Lizzi, F.A. Blood 36, 353-360 (1970)

4. Janossy, G., Roberts, M. \& Greaves, M.F. Lancet 2, 1058-1061 (1976).

5. Deininger, M.W., Goldman, J.M. \& Melo, J.V. Blood 96, 3343-3356 (2000)

6. He, Y. et al. Blood 99, 2957-2968 (2002).

7. Danhauser Riedl, S., Warmuth, M., Druker, B.J., Emmerich, B. \& Hallek, M. Cancer Res. 56 3589-3596 (1996).

8. Warmuth, M. et al. J. Biol. Chem. 272, 33260-33270 (1997)

9. Klejman, A. et al. EMBO J. 21, 5766-5774 (2002).

10. Donato, N.J. et al. Blood 101, 690-698 (2003).

11. Brumpt, C. et al. Blood 96, 2254-2261 (2000).

12. Saijo, K. et al. Nat. Immunol. 4, 274-279 (2003).

13. Roumiantsev, S., de Aos, I.E., Varticovski, L., Ilaria, R.L. \& Van Etten, R.A. Blood 97, 4-13 (2001).

14. Takahashi, N., Miura, I., Saitoh, K. \& Miura, A.B. Blood 92, 4758-4763 (1998).

\title{
The rat comes clean
}

\author{
Caroline A Wallace \& Timothy J Aitman
}

The rat genome sequence, the third mammalian genome sequence to be generated, was recently reported in Nature. The sequence provides new insights into mammalian genome evolution and the opportunity to translate decades of descriptive phenotyping to an understanding of the molecular mechanisms that underlie these phenotypes.

\section{The importance of being Rattus}

The rat has an important place in our social and demographic history (Fig. 1). Through its contributions to famine and the spread of fatal diseases, and its place in everyday language as a term of abuse, the rat has become synonymous with fear and loathing. Even in the scientific community, the value of the rat, as compared with the mouse, has been hotly debated, both in terms of its suitability as a model organism and its contribution to the bank of genome sequences now available. The

Caroline A. Wallace and Timothy J. Aitman are in the Physiological Genomics and Medicine Group, MRC Clinical Sciences Centre, Hammersmith Hospital, Imperial College London, UK.

e-mail:t.aitman@csc.mrc.ac.uk
Rat Genome Sequencing Project Consortium (RGSPC) has now published a high-quality draft sequence covering over $90 \%$ of the rat genome ${ }^{1}$. The rat genome sequence will yield new insights when it is laid alongside those of mice and humans and also promises to advance diverse areas of biology explored during 50 years of research in rat genetics, toxicology, pharmacology and physiology ${ }^{2}$.

Drawing on experience from sequencing the human, mouse and other genomes, the RGSPC adopted a combined BAC sequencing and whole-genome shotgun (WGS) strategy. From the outset, the intention was not to provide finished sequence, although at an estimated 1.32 errors per $10 \mathrm{~kb}$, the sequence quality is close to that accepted for finished sequence. The $2.75 \mathrm{~Gb}$ draft sequence has $7 \times$ coverage generated from 36 million high- quality sequence reads, $60 \%$ of which originated from WGS and 40\% from BACs. To combine the BAC and WGS sequence reads, the RGSPC developed the Atlas software package $^{3}$, which they also used to give users immediate access to BAC assemblies as each BAC was processed.

The completion of the draft sequence raises the questions of whether finished sequence should be generated for the rat and whether new sequence from other strains should be pursued. The rat community must also decide how the available sequence will be curated and error-checked in the future.

\section{Three's company}

The availability of a third mammalian genome sequence presents new opportunities for comparative genome analysis. In a three-way 
analysis of human, mouse and rat, the RGSPC identified an 'ancestral core' comprising 39\% of the euchromatic rat genome, which was under strong purifying selection across all three genomes. The ancestral core contains a high proportion (95\%) of all known coding exons and regulatory regions. In the conserved core, noncoding regions outnumber coding regions, suggesting that these noncoding, presumably regulatory elements have a crucial role in mammalian biology.

Segmental duplications were more common in the rat (occupying 3\% of the genome) than the mouse $(1-2 \%)$ but less common than in humans (5-6\%). As the RGSPC points out, this could be due in part to the different methods and states of completion of the three genome sequences. Analysis of the rat sequence showed evidence for two apparent evolutionary 'bursts', occurring around 5 and 8 million years ago. These may represent active periods of gene duplication within gene families in the rat genome.

Comparative analysis of the three genomes together enhanced the identification of consensus regulatory elements and splice signals and showed that mutation rates differed among mice, rats and humans. Comparative analysis also markedly increased the specificity with which transcription factor binding sites could be identified and, in particular, minimized false positive predictions arising in ancestral repeats. These analyses provide important insights into the biology of the three genomes and the ways in which the species evolved and diversified.

The theme of comparative analysis is elaborated and extended in the April issue of Genome Research, which is dedicated to articles on the biology of the rat genome. Nine articles compare rat, human and mouse sequence at the level of individual genes, chromosomal segments and the whole genome, with other articles on segmental duplication, comparative recombination rates and evolutionary hotspots.

\section{Whither now the rat?}

Before the rat genome sequencing project, the rat community had been quietly putting in place a foundation of rat genetics resources that would greatly enhance the utility of the genome sequence. For example, PhysGen, a Program for Genomics Applications funded by the US National Heart, Lung, and Blood Institute, created two panels of consomic strains for genetic dissection of disorders of the heart, kidney, lung

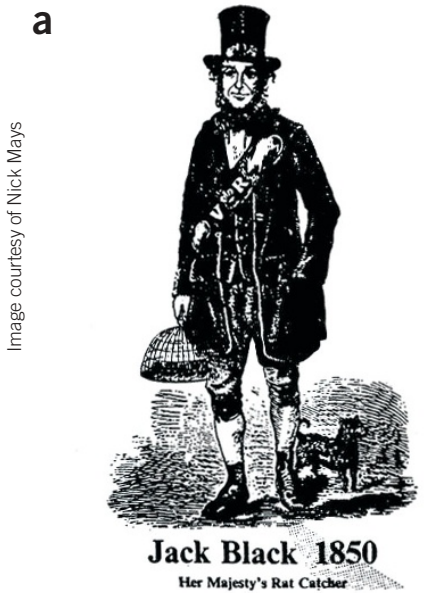

b

Figure 1 Historical rodent rivalries. (a) Jack Black was Queen Victoria's Royal Rat Catcher during the late 1800s. His leather belt was inset with cast-iron rats. When he caught any unusually colored rats, he bred them to establish new color varieties, which he sold to "well-bred young ladies". Perhaps he supplied rats to Mary Douglas, whose black and white hooded rat (b) won 'Best in Show' at the National Mouse Club exhibition in 1901. By 1912, there was enough interest in rats that the Club's name was officially changed to the National Mouse and Rat Club.

and vasculature. This is complemented by the Rat Genome Database, also funded by the US National Heart, Lung, and Blood Institute, which serves as a central repository for rat genetic and genomic data including dense genetic and radiation hybrid maps, over 20,000 genetic markers (now mapped onto the genome sequence), hundreds of mapped quantitative trait loci and virtual tools for comparative genetic analysis. In Europe, the reciprocal $\mathrm{HXB} / \mathrm{BXH}$ recombinant inbred strains, derived from the Spontaneously Hypertensive and Brown Norway rat strains, are one of the largest of all existing rodent recombinant inbred panels and one of the outstanding resources for genetic analysis of cardiovascular and metabolic phenotypes ${ }^{4,5}$. It is fortuitous that all of the consomic and recombinant inbred strains share, as one of their founder strains, the Brown Norway rat, from which the genome sequence was generated.

With these established resources and newly accumulating resources, such as large numbers of $\mathrm{cSNPs}^{6}$, what now is possible for the rat? In the next few years, we expect to see the successful positional cloning of substantial numbers of genes underlying complex rat phenotypes. Rat geneticists have been working to capture large numbers of these quantitative trait loci in congenic strains for many years. The potential for cloning these genes and for translating the results to the study of human disease was recently recognized by the
Wellcome Trust in awarding funds for cardiovascular functional genomics to a UK consortium of geneticists working on the genetics of hypertension, insulin resistance and type 2 diabetes in rats and humans.

But what of mechanism? The rat genetics community has long been bereft of the opportunity to test directly the function of rat genes and alleles and, although rat transgenesis and mutagenesis have been available and applied for more than a decade ${ }^{7-9}$, rat geneticists have had to turn to the mouse for knockout studies. Here, too, there is sign of a thaw. With the recent cloning of fertile adult rats using nuclear transfer ${ }^{10}$ comes the promise of rat knockouts to order, using homologous recombination and the new nuclear transfer protocol.

These are exciting times for rat biologiststhe opportunities for translating decades of descriptive rat phenotyping to an understanding of the molecular basis of these phenotypes are now there for the taking.

1. Rat Genome Sequencing Project Consortium. Nature 428, 493-521 (2004)

2. Jacob, H.J. \& Kwitek, A.E. Nat. Rev. Genet. 3, 33-42 (2002).

3. Havlak, P. et al. Genome Res. 14, 721-732 (2004).

4. Pravenec, M., Klir, P., Kren, V., Zicha, J. \& Kunes, J. J. Hypertens. 7, 217-221 (1989).

5. Pravenec, M. et al. Physiol. Res. (in the press).

6. Zimdahl, H. et al. Science 303, 807 (2004).

7. Mullins, J.J., Peters, J. \& Ganten, D. Nature 344, 541-544 (1990).

8. Zan, Y. et al. Nat. Biotechnol. 21, 645-651 (2003)

9. Pravenec, M. et al. Nat. Genet 27, 156-158 (2001).

10. Zhou, Q. et al. Science 302, 1179 (2003). 